\title{
OPTICAL SPECTROSCOPY OF BRIGHT FERMI LAT BLAZARS
}

\author{
Michael S. Shaw ${ }^{1}$, Roger W. Romani ${ }^{1}$, Stephen E. Healey $^{1}$, Garret Cotter $^{2}$, Peter F. Michelson $^{1}$, And \\ Anthony C. S. ReADHEAD ${ }^{3}$ \\ ${ }^{1}$ Department of Physics/KIPAC, Stanford University, Stanford, CA 94305, USA \\ 2 Department of Astrophysics, University of Oxford, Oxford OX1 3RH, UK \\ ${ }^{3}$ Department of Astronomy, California Institute of Technology, Pasadena, CA 91125 , USA \\ Received 2009 June 1; accepted 2009 August 25; published 2009 September 23
}

\begin{abstract}
We report on Hobby-Eberly Telescope and Palomar $5 \mathrm{~m}$ spectroscopy of recently identified $\gamma$-ray blazars in the Fermi LAT Bright Source List. These data provide identifications for 10 newly discovered $\gamma$-ray flat spectrum radio quasars and six new BL Lacertae (BL Lac) objects plus improved spectroscopy for six additional BL Lac objects. We substantially improve the identification completeness of the bright LAT blazars and give new redshifts and $z$ constraints, new estimates of the black hole masses, and new measurements of the optical spectral energy distribution.
\end{abstract}

Key words: BL Lacertae objects: general - galaxies: active - quasars: general - surveys

Online-only material: color figure

\section{INTRODUCTION}

The Fermi Large Area Telescope (LAT) pair production telescope has been surveying the $20 \mathrm{MeV}-300 \mathrm{GeV} \gamma$-ray sky since 2008 August 11. Among the many sources being detected by this mission, blazars dominate the extragalactic sky. The first published set of these objects, the "LAT Bright AGN Sample" (LBAS) based on three months of sky survey exposure, included 132 bright $(\geqslant 10 \sigma)$ detections at $|b|>10^{\circ}$. The bulk (117) of these sources have been associated with flat spectrum radio counterparts, which in turn end up being mostly well-known flat spectrum radio quasars (FSRQ) and BL Lacertae (BL Lac) objects. A few additional sources are pulsars and a few remain unidentified. The associations are based on the distribution of radio and $\mathrm{X}$-ray properties of the radio counterparts (CRATES; Healey et al. 2007), and the analysis allows a quantitative assessment of the probability of association.

Even before the start of the Fermi mission, it was recognized that a large sample of these blazars would be needed for source identification; in the Candidate Gamma-Ray Blazar Survey (CGRaBS; Healey et al. 2008, and references therein), we pursued optical spectroscopy of the sources selected to be most similar to the previously known EGRET (Energetic Gamma Ray Experiment Telescope) blazars. This set of 1625 blazars included many sources without previous spectroscopic identification. Here we report on previously unpublished optical spectra from our CRATES/CGRaBS survey and observations for new sources from the LBAS list. The data allow the classification of the blazar type and redshift solutions for the strong-lined objects. Significant redshift constraints are also obtained for the weak-lined BL Lac objects. These measurements contribute 10 FSRQ redshifts, one new spectroscopic redshift for a BL Lac object, and 11 additional BL Lac object redshift constraints. Including these new data, $91 \%$ of all LBAS sources have been spectroscopically typed, $84 \%$ have redshifts, and $70 \%$ of the BL Lac objects have spectroscopic redshifts. We also use our spectra to examine the black hole $(\mathrm{BH})$ masses and optical continua of these blazar sources.

In this paper, we assume an approximate concordance cosmology $-\Omega_{m}=0.3, \Omega_{\Lambda}=0.7$, and $H_{0}=70 \mathrm{~km} \mathrm{~s}^{-1} \mathrm{Mpc}^{-1}$.

\section{OBSERVATIONS AND DATA ANALYSIS}

\subsection{HET}

The bulk of the spectroscopic observations were performed with the $9.2 \mathrm{~m}$ Hobby-Eberly Telescope (HET) at McDonald Observatory; many of these observations were part of the CGRaBS survey (Healey et al. 2008), but additional data were taken on newly discovered LBAS sources. The HET observes in the declination range $-11^{\circ}<\delta<+73^{\circ}$, and is fully queue scheduled (Shetrone et al. 2007), allowing us to receive data remotely year round and to spread the cost of inclement weather and unfavorable conditions among the observing programs. We use the Marcario Low-Resolution Spectrograph (LRS; Hill et al. 1998), with grism G1 (300 lines $\mathrm{mm}^{-1}$ ), a $2^{\prime \prime}$ slit, and a Schott GG385 long-pass filter for a resolution of $R \approx 500$ between $4150 \AA$ and $10500 \AA$. Typical exposures are $2 \times 600 \mathrm{~s}$ for FSRQ objects and $2 \times 900 \mathrm{~s}$ for BL Lac objects, with the slit placed along the parallactic angle.

\subsection{Double Spectrograph}

We also used the double spectrograph (DBSP) on the $5 \mathrm{~m}$ Hale Telescope at Palomar. The observations with a $1^{\prime \prime}$ slit at the parallactic angle used a 600 line $\mathrm{mm}^{-1}$ grating on the blue side, covering $\lambda \lambda 3100-5200$ at a resolution of $\sim 2.8 \AA$ $(R \sim 1450)$. The red camera, with a 316 line $\mathrm{mm}^{-1}$ grating covers $\lambda \lambda 5200-9500$, at a resolution $\sim 5.2 \AA(R \sim 1350)$. P200/DBSP is our primary tool for targets outside of the HET declination range (i.e., $-35^{\circ}<\delta<-11^{\circ}$ and $+73^{\circ}<\delta$ ) and provides extra spectral coverage and resolution in the blue for bright BL Lac objects. Typical exposures were $2 \times 600 \mathrm{~s}$ for FSRQs and up to $3 \times 900$ s for faint BL Lac objects.

\subsection{Analysis Pipeline}

Data reduction was performed with the IRAF package (Tody 1986) using standard techniques. Wavelength calibration was performed with a neon-argon lamp at the HET and on the red side at the DBSP, and iron-argon on the DBSP blue side. For these relatively faint objects, we employ an optimal extraction algorithm (Valdes 1992) to maximize the $\mathrm{S} / \mathrm{N}$. 
We perform spectrophotometric calibration using standard stars from Oke (1990). In most cases, the standard exposures from the data night were suitable, but at the HET, standards from subsequent nights were sometimes used. Due to differential slit losses and variable conditions between object and standard star exposures, we estimate that the accuracy of our absolute spectrophotometry is $\sim 30 \%$ (Healey et al. 2008), although the relative spectrophotometry is considerably better. Spectra are corrected for telluric absorptions and visually cleaned of cosmic rays. Multiple exposures on a single target are combined into a single spectrum, weighting by $\mathrm{S} / \mathrm{N}$.

We visually identify and measure emission line equivalent widths and full widths at half-maxima (FWHMs) and derive redshifts from these line measurements. They are listed in Table 1. Line and redshift measurement techniques are discussed in Section 3.1.

\section{RESULTS}

We present spectra for 10 FSRQ objects, six new BL Lac objects' identifications and spectra, and high S/N spectroscopy on six additional objects previously known to be BL Lac objects. We present spectroscopic redshifts for all $10 \mathrm{FSRQ}$, and for one BL Lac object. We derive virial BH masses for the FSRQ, and for BL Lac objects that lack spectroscopic redshifts, we extract significant constraints on the redshift.

\subsection{FSRQ Spectra}

We present 10 previously unpublished FSRQ spectra (Figure 1) with redshifts ranging from 0.227 to 1.805 . These redshifts were previously listed in Abdo et al. (2009). All redshifts are confirmed by multiple emission lines and derived by cross-correlation analysis using the RVSAO package (Kurtz \& Mink 1998). Table 1 gives the approximate continuum fluxes at $10^{14.7} \mathrm{~Hz}(\lambda=5981 \AA)$ and spectral indices $\alpha\left(F_{v} \propto v^{-\alpha}\right)$. The fluxes, as above, have uncertainties as large as $30 \%$ due to unknown slit losses. The spectral index uncertainties are estimated by fits to independent subsets of the spectral range.

The properties of strong emission lines are listed in Table 1. We are particularly interested in the broad emission lines $\mathrm{H} \beta$, $\mathrm{Mg}$ II, and $\mathrm{C}$ IV, as their virialized broad components are used to make estimates of the $\mathrm{BH}$ mass. For these lines, we take care to measure the Gaussian FWHM of the broad component, avoiding contamination by other spectral features.

For $\mathrm{H} \beta$, it is important to include narrow components in the line fit. We fit the continuum to a power law, and, following McLure \& Dunlop (2004), we simultaneously fit broad and narrow $\mathrm{H} \beta$, narrow [O III]4959 $\AA$, and narrow [O III]5007 $\AA$. We require rest wavelengths of narrow $\mathrm{H} \beta$ and the [O III] lines to match laboratory values; the broad $\mathrm{H} \beta$ wavelength is free. All lines are modeled with Gaussian profiles. The continuum is measured at $5100 \AA$.

For $\mathrm{Mg}$ II, we first subtract off our power-law fit. Then, we subtract a template (Tsuzuki et al. 2006) of the broad Fe II complexes near $2800 \AA$ to minimize contamination with $\mathrm{Mg}$ II. We then fit the Mg II line itself with a broad+narrow Gaussian. For this line, the continuum measurement is, as usual, made at $3000 \AA$, to avoid Fe contamination (McLure \& Dunlop 2004).

$\mathrm{C}$ IV lines sometimes suffer strong associated absorption, but such corrections were modest for the few cases presented here. Also, for consistency with the Sloan Digital Sky Survey (SDSS) measurements (see below) and to minimize sensitivity to associated absorbers, we report here the FWHM of the actual C IV line (Shen et al. 2008).

\subsection{Black Hole Mass Estimates}

We can use the emission line measurements reported above to estimate $\mathrm{BH}$ masses for the FSRQ objects in our sample using the empirical "virial" scaling relationships (McLure \& Jarvis 2002). Mass scaling relations have been applied to large samples of quasars in the past and are calibrated by reverberation mapping techniques (Vestergaard \& Osmer 2009).

Continuum flux measurements were made for each line at the appropriate rest wavelength $(5100 \AA$ for $\mathrm{H} \beta, 3000 \AA$ for $\mathrm{Mg}$ II, $1350 \AA$ for C IV), and converted to continuum luminosity $\lambda L_{\lambda}$, using our assumed cosmology. For two objects in our sample, $\mathrm{J} 1012+2439$ and $\mathrm{J} 2327+0940$, the C IV line is clearly present, but the spectra do not extend blueward enough to cover $1350 \AA$; for these, we extrapolate a power-law fit to the continuum at longer wavelengths. We follow Shen et al. (2008) in the calculation of the $\mathrm{BH}$ masses:

$$
\log \left(\frac{M}{M_{\odot}}\right)=a+b \cdot \log \left(\frac{\lambda L_{\lambda}}{10^{44} \mathrm{erg} \mathrm{s}^{-1}}\right)+2 \log \left(\frac{\mathrm{FWHM}}{\mathrm{km} \mathrm{s}^{-1}}\right)
$$

where $a=0.505, b=0.62$ for $\mathrm{Mg}$ II; $a=0.672, b=0.61$ for $\mathrm{H} \beta$; and $a=0.66, b=0.53$ for C IV.

Masses are plotted in Figure 2. Values measured from spectra presented in this paper are indicated by filled points (species used indicated by point style). Some of the LBAS FSRQ have spectra in the SDSS archive (Schneider et al. 2007). We recovered these and measured line profiles and continuum fluxes exactly as for our new objects to obtain similar BH mass estimates (open points). For about half of these sources, the SDSS published independently estimated values for $M$ (Shen et al. 2008). We find adequate agreement, with rms differences in $\log (M)$ of $0.04(\mathrm{H} \beta)$ and 0.14 (Mg II, C IV), comparable to the interspecies dispersion.

In general, the virial mass estimators are of limited accuracy for individual objects. As one illustration, when estimates are available for a given object from more than one species, we connect the points with a dashed line. The pipeline processing of the entire Sloan DR5 sample of optically selected quasars provides a large number of such mass estimates. In Figure 2, we show the mean and $\mathrm{rms} \log (M)$ values as a function of redshift. For both the $\gamma$-ray-selected sample presented here and the optical SDSS sample, the mean mass increases with redshift, likely a simple flux bias. However, we note that the $\gamma$-ray sources trend about $3 \times$ lower in mass than the optically selected objects, albeit with large scatter. There are several possible origins of this trend. One possibility is that this is a result of the fainter optical limit in the follow-up to the LBAS sample. Another selection effect may be imposed by the $\gamma$-ray selection's dependence on the jet flux, which, as a strong function of the viewing angle to the relativistic jet, is more weakly dependent on the underlying source luminosity and presumably BH mass. A final possibility stems from orientation: virial estimates (Equation (1)) assume isotropic velocities in the broad line region. However, blazars are known to have the jet axis, and hence the $\mathrm{BH}$ spin and inner accretion disk axis, close to the Earth line of sight. If the broad line region has a toroidal structure (Fine et al. 2008), then blazars will have lines with FWHM decreased by $\langle\sin i\rangle$ and lower virial mass estimates than quasi-stellar objects (QSOs) viewed at larger inclination angles. Larger data sets from future LAT samples should allow us to probe the reality and origin of such effects. 
Table 1

Line Properties for New LBAS FSRQ

\begin{tabular}{|c|c|c|c|c|c|c|c|c|c|c|c|c|c|}
\hline \multirow[t]{2}{*}{ Fermi Name } & \multirow[t]{2}{*}{ Name } & \multirow[t]{2}{*}{ R.A. } & \multirow[t]{2}{*}{ Decl. } & \multirow{2}{*}{\multicolumn{2}{|c|}{$\begin{array}{l}F_{v, 10^{14.7}} \alpha \\
\operatorname{rg~cm}^{-2} \mathrm{~s}^{-1} \mathrm{~Hz}^{-1}\end{array}$}} & \multirow[t]{2}{*}{$z$} & \multicolumn{2}{|c|}{$\mathrm{H} \beta$} & \multicolumn{2}{|c|}{$\mathrm{Mg}$ II } & \multicolumn{2}{|c|}{ Civ } & \multirow[t]{2}{*}{ Other Lines } \\
\hline & & & & & & & $\begin{array}{l}\text { EW } \\
\AA_{\text {obs }} \\
\end{array}$ & $\begin{array}{l}\text { FWHM } \\
\mathrm{km} \mathrm{s}_{\text {rest }}^{-1} \\
\end{array}$ & $\begin{array}{l}\text { EW } \\
\AA_{\text {obs }} \\
\end{array}$ & $\begin{array}{l}\text { FWHM } \\
\mathrm{km} \mathrm{s}_{\text {rest }}^{-1} \\
\end{array}$ & $\begin{array}{l}\text { EW } \\
\AA_{\text {obs }} \\
\end{array}$ & $\begin{array}{l}\text { FWHM } \\
\mathrm{km} \mathrm{s}_{\text {rest }}^{-1} \\
\end{array}$ & \\
\hline OFGL J0017.4-0503 & J0017-0512 & 001735.8 & -051241.6 & 85 & $0.78 \pm 0.06$ & 0.227 & 59 & 3009 & & & & & $\mathrm{H} \alpha, \mathrm{H} \gamma, \mathrm{H} \delta, \mathrm{O}$ II, $\mathrm{O}_{\mathrm{III}}$ \\
\hline OFGL J0714.2+1934 & J0713+1935 & 071355.6 & 193500.3 & 13 & $1.3 \pm 0.07$ & 0.540 & 19 & 2815 & 30 & 2841 & & & $\mathrm{H} \alpha, \mathrm{H} \gamma, \mathrm{H} \delta, \mathrm{O}$ II, $\mathrm{O}_{\mathrm{III}}$ \\
\hline OFGL J1012.9+2435 & $\mathrm{J} 1012+2439$ & 101241.3 & 243923.3 & 4.6 & $1.0 \pm 0.05$ & 1.805 & & & 24 & 2489 & 45 & 2360 & C III, 1650,1730 \\
\hline OFGL J1034.0+6051 & $\mathrm{J} 1032+6051$ & 103253.9 & 605127.5 & 1.5 & $1.3 \pm 0.12$ & 1.064 & & & 140 & 6495 & & & \\
\hline OFGL J1333.3+5088 & $\mathrm{J} 1333+5057$ & 133353.7 & 505735.9 & 2.3 & $1.9 \pm 0.09$ & 1.362 & & & 28 & 3147 & & & $\mathrm{C}_{\text {III }}$ \\
\hline OFGL J1522.2+3143 & $\mathrm{J} 1522+3144$ & 152209.9 & 314414.3 & 4.3 & $1.8 \pm 0.18$ & 1.487 & & & 40 & 4086 & & & C III \\
\hline OFGL J1847.8+3223 & $\mathrm{J} 1848+3219$ & 184822 & 321902.6 & 7.5 & $0.71 \pm 0.16$ & 0.798 & 75 & 2878 & 52 & 2682 & & & $\mathrm{C}_{\mathrm{II}}$ \\
\hline OFGL J2157.5+3125 & $\mathrm{J} 2157+3127$ & 215728.8 & 312701.3 & 1.4 & $1.5 \pm 0.60$ & 1.486 & & & 103 & 4185 & & & $\mathrm{C}_{\text {III }}$ \\
\hline OFGL J2327.3+0947 & $\mathrm{J} 2327+0940$ & 232733.5 & 094009.5 & 16 & $0.51 \pm 0.07$ & 1.843 & & & 32 & 4840 & 24 & 6580 & C II, C III \\
\hline OFGL J2345.5-1559 & $\mathrm{J} 2345-1555$ & 234512.4 & -155507.8 & 31 & $1.3 \pm 0.02$ & 0.621 & 17 & 3835 & 17 & 3630 & & & $\mathrm{O}$ III, $\mathrm{H} \gamma, \mathrm{H} \delta$ \\
\hline
\end{tabular}




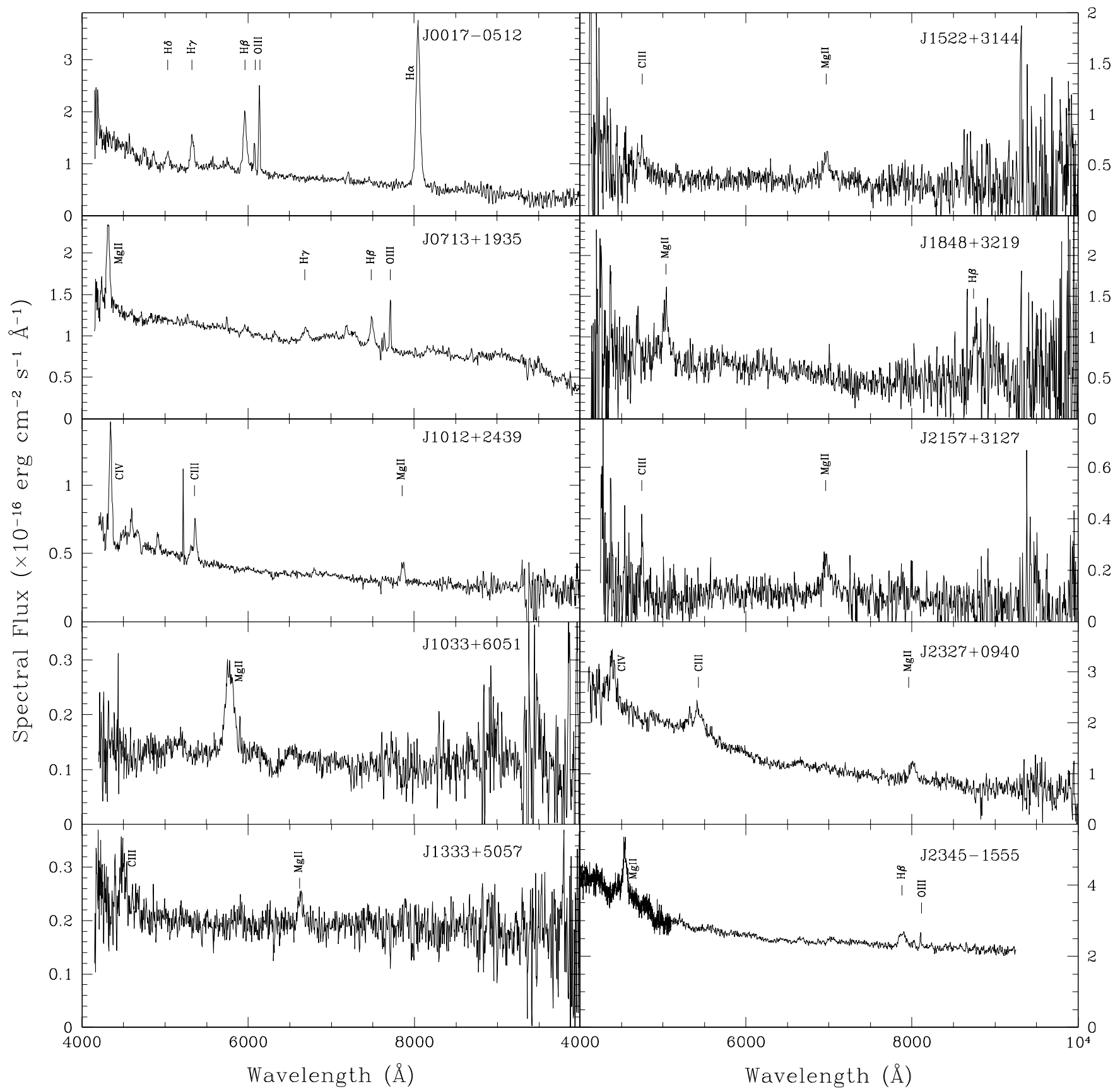

Figure 1. New spectra of FSRQ objects in the Fermi LBAS sample. The emission line properties are listed in Table 1.

\subsection{BL Lac Object Properties}

We have obtained high sensitivity $(\mathrm{S} / \mathrm{N} \sim 100)$ spectra of 12 LBAS BL Lac objects. Following Marcha et al. (1996) and Healey et al. (2008), we adopt here the pragmatic "optical spectroscopic" definition of a BL Lac object as a blazar lacking emission lines with observed equivalent width greater than $5 \AA$ and a limit on any possible $4000 \AA$ spectral break of $<40 \%$. For some sources in CGRaBS, we have seen that, as the continuum level fluctuates, the source passes from spectroscopic BL Lac object to spectroscopic FSRQ. We designate a source as a BL Lac object if during any of our observations with an adequate spectral range it has satisfied the spectroscopic definition. In this connection, one of the high-mass objects appearing in Figure 2 should be mentioned. This is J1058+0133, designated an FSRQ in LBAS based on a clear detection of a broad $\mathrm{Mg}$ II line at $z=$ 0.888 . However, this line has an EW of only $3.5 \AA$; accordingly, this blazar should be formally designated a BL Lac object at the epoch of the SDSS spectrum. The very strong non-thermal continuum, in fact, drives up the apparent luminosity and the $\mathrm{BH}$ mass estimate. This emphasizes that non-thermal continuum can artificially increase the apparent mass; the virial estimates are not calibrated for BL Lac object sources.

The spectral range requirement for BL Lac object's designation is that the spectrum covers $\left[\lambda_{\min }\right.$ to $\left.\lambda_{\max }\right]$ with sufficient $\mathrm{S} / \mathrm{N}$ to detect at least one of the standard active galactic nuclei (AGNs) broad emission lines: $\mathrm{Ly} \alpha, \mathrm{C}$ IV, $\mathrm{C}$ III, $\mathrm{Mg}$ II, $\mathrm{H} \beta$, or $\mathrm{H} \alpha$. In practice, this means adequate $\mathrm{S} / \mathrm{N}$ over $\lambda_{\max } / \lambda_{\min }>1.75$; this is easily satisfied for the BL Lac objects in our LBAS sample. 
Table 2

BL Lac Object Continuum Properties for LBAS Sources

\begin{tabular}{|c|c|c|c|c|c|c|}
\hline \multirow{2}{*}{ Fermi Name } & \multirow[t]{2}{*}{ Name } & \multirow{2}{*}{\multicolumn{4}{|c|}{ Decl. $\underset{10^{-28} \mathrm{erg} \mathrm{cm}^{-2} \mathrm{~s}^{-1} \mathrm{~Hz}^{-1}}{\mathrm{erg}^{14.7}} \alpha$}} & \multirow{2}{*}{$z$} \\
\hline & & & & & & \\
\hline OFGL J0050.5-0928 & J0050-0929 & 005041.3 & -09295.1 & 69 & $0.48 \pm 0.01$ & $>0.44$ \\
\hline OFGL J0112.1+2247 & $\mathrm{J} 0112+2244$ & 011205.8 & 224438.8 & 270 & $0.54 \pm 0.01$ & $>0.24$ \\
\hline OFGL J0144.5+2709 & J0144+2705 & 014433.5 & 270503.0 & 17 & $3.6 \pm 0.06$ & $>0.45$ \\
\hline OFGL J0712.9+5034 & $\mathrm{J} 0712+5033$ & 071243.6 & 503322.6 & 71 & $0.56 \pm 0.02$ & $>0.47$ \\
\hline OFGL J0722.0+7120 & $\mathrm{J} 0721+7120$ & 072153.4 & 712036.4 & 590 & $0.31 \pm 0.02$ & $>0.06$ \\
\hline OFGL J0909.7+0145 & J0909+0200 & 090939.85 & 020005.3 & 33 & $1.1 \pm 0.03$ & $>0.54$ \\
\hline OFGL J1054.5+2212 & $\mathrm{J} 1054+2210$ & 105430.6 & 221054.8 & 46 & $0.24 \pm 0.02$ & $>0.60^{\mathrm{a}}$ \\
\hline OFGL J1253.4+5300 & $\mathrm{J} 1253+5301$ & 125311.9 & 530111.6 & 42 & $0.56 \pm 0.01$ & $>0.77$ \\
\hline OFGL J1427.1+2347 & $\mathrm{J} 1427+2347$ & 142700.3 & 234759.9 & 600 & $0.01 \pm 0.01$ & $>0.03$ \\
\hline OFGL J1543.1+6130 & $\mathrm{J} 1542+6129$ & 154256.9 & 612955.3 & 61 & $0.74 \pm 0.02$ & $>0.63$ \\
\hline OFGL J1719.3+1746 & $\mathrm{J} 1719+1745$ & 171913 & 174506.3 & 25 & $0.67 \pm 0.03$ & $>0.58$ \\
\hline OFGL J2325.3+3959 & $\mathrm{J} 2325+3957$ & 232517.8 & 395736.5 & 17 & $0.76 \pm 0.02$ & $>1.05$ \\
\hline
\end{tabular}

Note.

${ }^{\mathrm{a}}$ Measured redshift $z=0.634$.

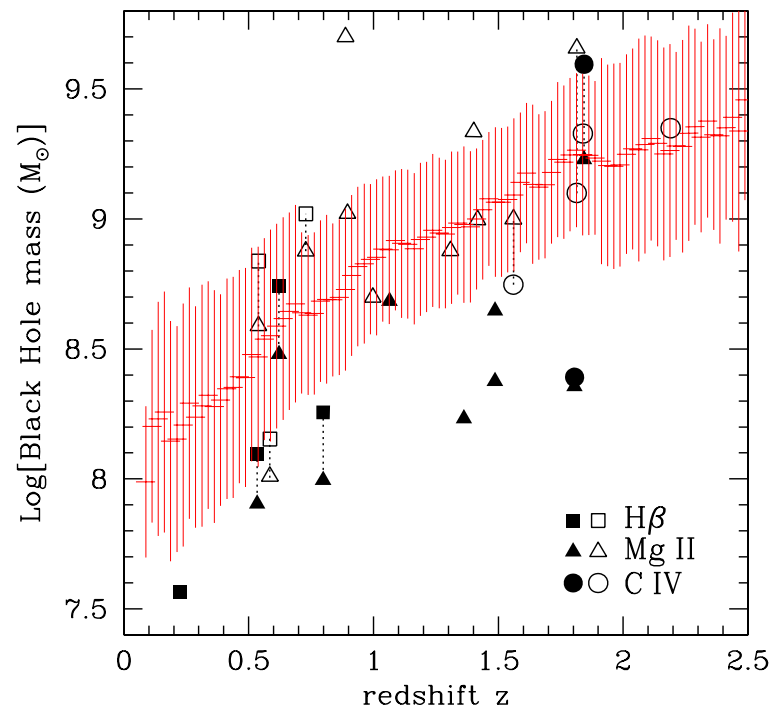

Figure 2. BH mass estimates as a function of redshift for LBAS sources. Filled points are measured from FSRQ spectra presented in this paper, open points are for spectra of LBAS FSRQ available from the SDSS archive (Squares: $\mathrm{H} \beta$ $\lambda$ 4861, Triangles: Mg II $\lambda 2800$, Circles: C IV $\lambda$ 1550). When two species are used for a given source, dashed lines connect the mass estimates. For comparison, the mean and rms range of mass estimates from the full SDSS catalog (Shen et al. 2008) are shown.

(A color version of this figure is available in the online journal.)

To describe the BL Lac object continua, we fit the spectra to a power law, using the IRAF nfit1d routine. Most are well fit with rising (blue) power laws, suggesting a synchrotron $v F_{v}$ peak well above the optical band. J0144+2705 is, however, very red. This is confirmed by USNO B1/2MASS fluxes which rise to the $J$ band and flatten above $1.5 \mu \mathrm{m}$. The Galactic extinction in the direction of this source is $A_{B}=0.3 \mathrm{mag}$, so local absorption is unlikely to redden the source. It seems plausible that this BL Lac object has a low synchrotron peak frequency $v_{\max } \sim 10^{14} \mathrm{~Hz}$. This may be tested by broader study of the spectral energy distribution (SED).

The spectral indices $\left(F_{v} \propto v^{-\alpha}\right)$ and fluxes of the BL Lac object are reported in Table 2 . For the continuum normalization, we assume the $30 \%$ absolute spectrophotometric uncertainty noted above; for the spectral indices, we again estimate the dominant systematic errors from fits to non-overlapping sections of the continuum. The continuum-normalized spectra are shown in Figures 3 and 4. These plots also label notable interstellar features e.g., $\mathrm{Na} \lambda 5892$, and the diffuse interstellar bands (DIBs; Herbig 1995).

\subsection{BL Lac Object Redshift Limits}

The strong dominance of the non-thermal nuclear continuum in BL Lac objects guarantees that the equivalent widths of any broad nuclear lines or absorption features from the host galaxy are very small. While recent work with $8 \mathrm{~m}$-class telescopes has provided some redshift solutions, spectroscopic redshifts for many objects remain elusive (Sbarufatti et al. 2005). Nevertheless, quantitative limits on host spectral features can be useful in extracting redshift constraints. For example, based on the lack of a Ly $\alpha$ forest-induced break in the HET spectra, none of our BL Lac objects can have $z>2.41$. This rather weak bound can be improved with UV coverage beyond the HET LRS 4150 Å limit.

Also, there is good evidence, especially from Hubble Space Telescope imaging, that $\mathrm{BL}$ Lac objects reside in relatively uniform giant elliptical hosts, with $M \sim-22.9$ (Sbarufatti et al. 2005). Analysis to limit the contribution of such a host to the observed spectrum can thus provide a lower limit on the BL Lac object redshift (Sbarufatti et al. 2006). We develop here a similar spectral bound on redshift, using the $\mathrm{Ca} \mathrm{H} / \mathrm{K}$ and $G$-band absorption features, the strongest narrow features in the host spectrum.

We first determine the expected equivalent width of a given absorption line by measuring an elliptical template spectrum and then computing the net equivalent width in the observed host+nucleus spectrum at each redshift following Sbarufatti et al. (2006):

$$
\mathrm{EW}_{\text {expected }}=\frac{(1+z) \mathrm{EW}_{h}}{1+F_{n} / F_{h}}
$$

where $\mathrm{EW}_{h}$ is the equivalent width of the line in the host galaxy spectrum, $F_{n}$ is the nuclear flux at the wavelength of that absorption line, and $F_{h}$ is the flux of the giant elliptical host at that wavelength. $F_{h}$ is corrected for the slit/extraction aperture losses, assuming a deVaucoleurs profile with the redshiftdependent angular size set by a constant radius $r_{0}=10 \mathrm{kpc} . F_{n}$ is the observed flux minus that expected from the host in our extraction aperture. This predicted feature strength is computed for the major absorption lines at each redshift. 


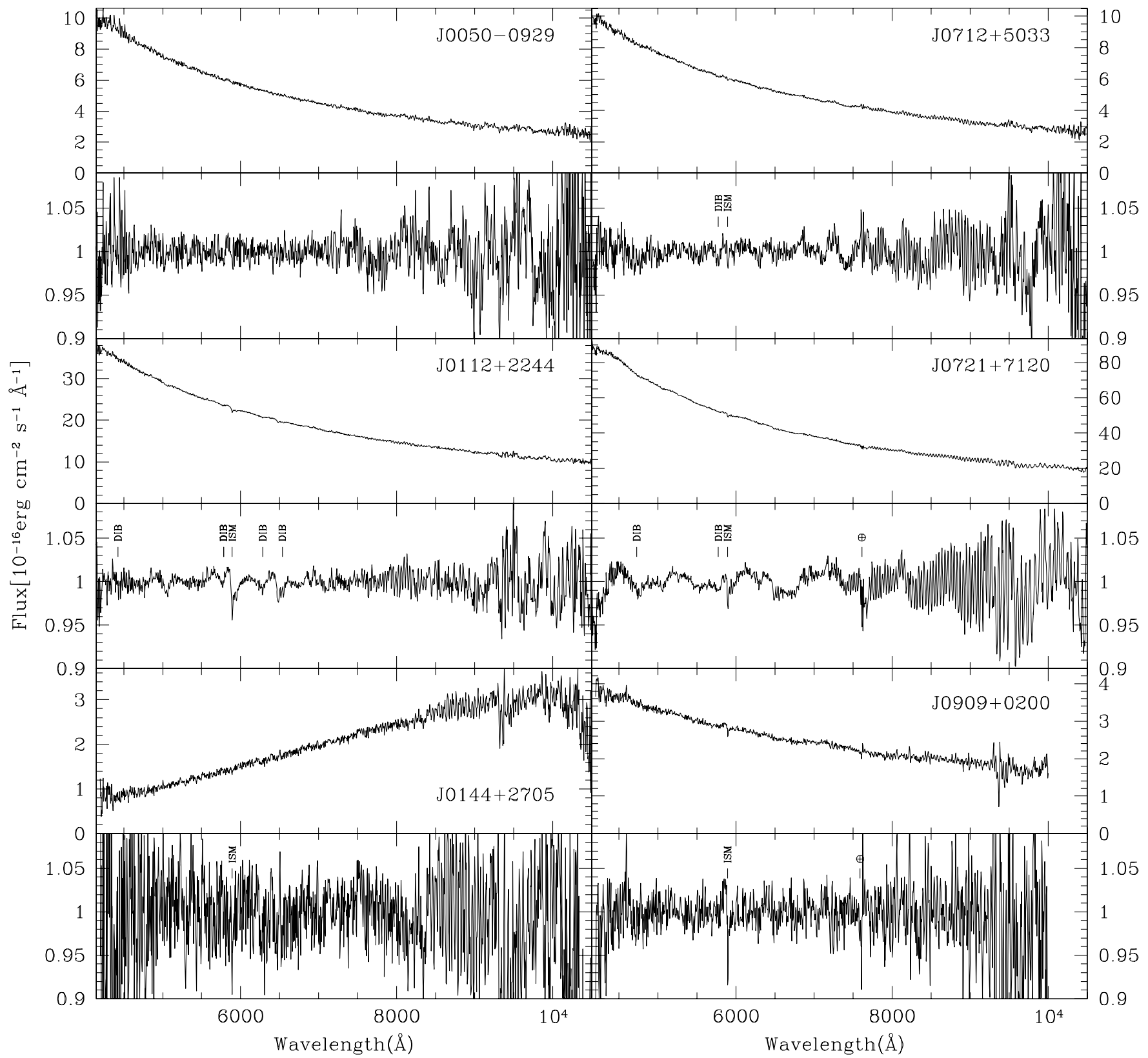

Figure 3. New spectra of LBAS BL Lac objects. Top panels are flux-calibrated spectra with flux in $10^{-16} \mathrm{erg} \mathrm{cm}^{-2} \mathrm{~s}^{-1} \AA^{-1}$. Bottom panels show spectra normalized by dividing out the continua. Telluric and interstellar absorption features are labeled.

We next compute the $3 \sigma$ limit on narrow absorption features in the observed spectrum as a function of wavelength from the local rms of negative spectral fluctuations, after highpass filtering to remove broad continuum features and after exclusion of zero-redshift Galactic and telluric features. We find that the line sensitivity varies considerably across the spectrum (Sbarufatti et al. 2006 assume constant sensitivity for their VLT spectra). For the HET data, limited blue sensitivity weakens the constraints below $\sim 5000 \AA$, while strong fringing (uncorrectable due the varying HET pupil) weakens the bounds beyond $\sim 8000 \AA$. The sensitivity also varies due to the changing resolution; this is especially important for the DBSP data, although no DBSP spectra are used in the measurements of the BL Lac objects presented here.

Comparison of the expected and limiting EW curves gives a lower limit on the BL Lac object redshift (for the assumed standard host magnitude) whenever one absorption feature is excluded at the $3 \sigma$ level. Generally, the Ca features provide the strongest constraint. However, since our HET spectra are limited to $\lambda>4150 \AA$, the $G$ band at $\lambda=4304 \AA$ is used to exclude $z \leqslant 0.05$. Figure 5 shows the exclusion curve for J1253+5301, where we find $z>0.77$. The results for the BL Lac objects data are presented in Table 2. With our HET data, we can exclude redshifts less than $z \sim 0.5-0.8$. For a few particularly bright BL Lac object, however, the redshift constraints are substantially weaker as the expected equivalent widths are smaller. Higher resolution and higher $\mathrm{S} / \mathrm{N}$ spectra can, of course, improve these bounds.

A few objects deserve particular comment. For J1054+2210, after completing the redshift limit analysis, weak $\mathrm{H} / \mathrm{K}$ and $G$-band features plus O II $\lambda 3727$ emission were found at redshift $z=0.634$. This detection is confirmed by cross-correlation 


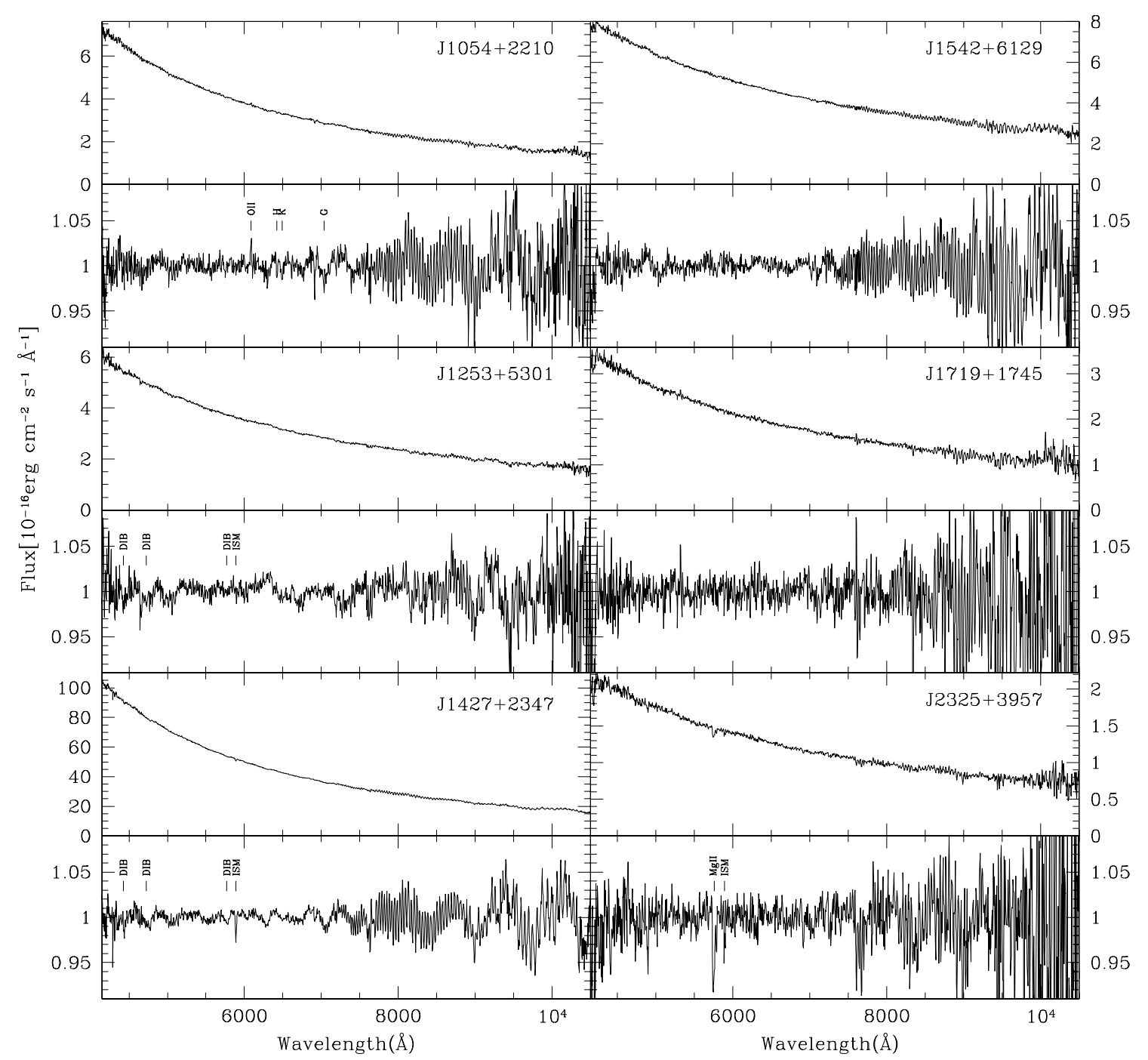

Figure 4. As for Figure 3. J1054+2210 has a redshift solution $z=0.634$. J2325+3957 has an intervening absorption line system at $z=1.04$.

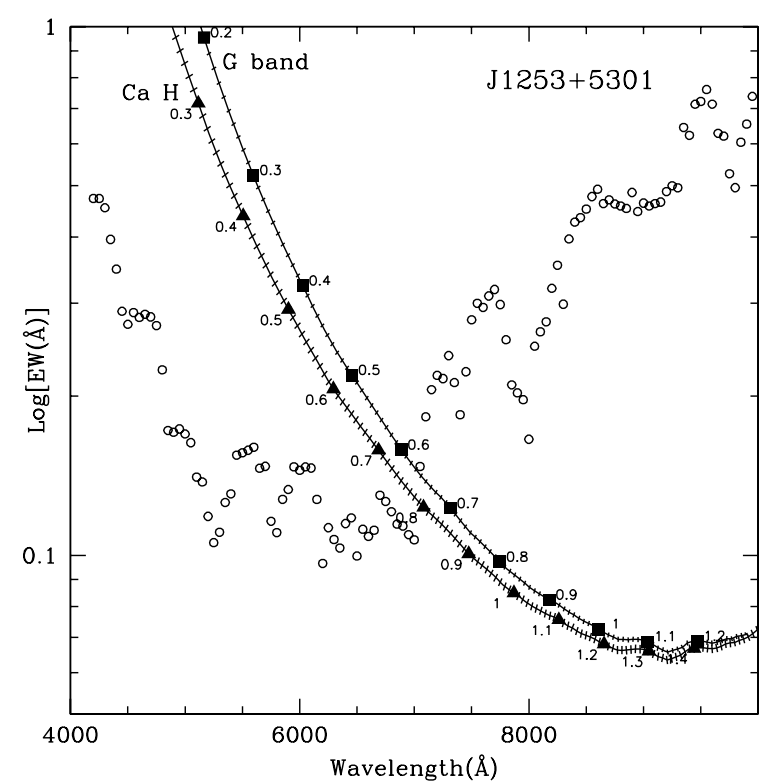

Figure 5. Expected equivalent widths of the $H(3934 \AA$, triangles) and $G(4304 \AA$, squares) features calculated at different redshifts. The $3 \sigma$ limit on line equivalent widths across the spectrum is plotted with open circles. As long as either $\mathrm{H}$ or $\mathrm{G}$ is above the circles, the corresponding redshift is excluded. Here, we excluded $z<0.77$. analysis. The measured redshift is just above the redshift bound of $z>0.60$ given by the exclusion analysis. For J2325+3957, the EW limit analysis gave a redshift bound of $z>0.77$. We have detected an intergalactic absorption system in this spectrum, with $\mathrm{Mg}$ II doublet and Fe II absorption features corresponding to $z=1.04$. This stronger bound, listed in Table 2, is consistent with the EW limit analysis.

\section{CONCLUSIONS}

The optical spectroscopy presented here provides significant new information on some of the less well-known members of the set of the LBAS $\gamma$-ray blazars.

For all of the new FSRQ, precision redshifts are measured from the spectra. For one of the new BL Lac objects, we determine the redshift spectroscopically; for the others, we extract limits on the redshift from the spectrum. Continuum fluxes and spectral indices are provided that will be useful for SED plots. These measurements bring the LBAS to $84 \%$ redshift completion. For most of the unsolved BL Lac objects, we extract significant lower limits on the redshift. These will be helpful in modeling the $\gamma$-ray BL Lac object population and evolution and in focusing redshift searches to a narrower spectral range.

For the strong-line objects, we present estimates of the virial $\mathrm{BH}$ masses. Although such mass estimates have large 
uncertainties, our objects appear to have somewhat lower mass than typical for the SDSS quasar sample. Like that sample, the mean mass increases with redshift. In the one case where a BL Lac object-like object has a mass measurement, the excess continuum artificially inflates the apparent $\mathrm{BH}$ mass.

The spectroscopic analysis presented here for the small LBAS blazar sample will be much more powerful when applied to the full sample of LAT blazars detected during the first year sky survey, which should include 500-1000 objects. We continue to collect spectroscopic data on the LAT blazars as they are detected and plan to use the optical analysis of this larger data set to probe the evolution of the $\gamma$-ray-emitting objects.

The HET is a joint project of the University of Texas at Austin, the Pennsylvania State University, Stanford University, Ludwig-Maximilians-Universitaet Muenchen, and GeorgAugust-Universitaet Goettingen. The HET is named in honor of its principal benefactors, William P. Hobby and Robert E. Eberly. The Marcario Low Resolution Spectrograph is named for Mike Marcario of High Lonesome Optics, who fabricated several optics for the instrument but died before its completion. The LRS is a joint project of the Hobby*Eberly Telescope partnership and the Instituto de Astronoma de la Universidad Nacional Autonoma de Mexico.
M.S.S. and S.E.H. are supported by NASA under grants NNX08AW30G and NAS5-00147. A.C.S.R. is supported by the NSF under grant AST-0808050.

\section{REFERENCES}

Abdo, et al. 2009, ApJ, 700, 597

Fine, S., et al. 2008, MNRAS, 390, 1413

Healey, S. E., et al. 2007, ApJS, 171, 61

Healey, S. E., et al. 2008, ApJS, 175, 97

Herbig, G. H. 1995, ARA\&A, 33, 19

Hill, G. J., Nicklas, H. E., MacQueen, P. J., Tejada, C., Cobos Duenas, F. J., \& Mitsch, W. 1998, Proc. SPIE, 3355, 375

Kurtz, M. J., \& Mink, D. J. 1998, PASP, 110, 934

Marcha, M.J.M., et al. 1996, MNRAS, 281, 425

McLure, R. J., \& Dunlop, J. S. 2004, MNRAS, 352, 1390

McLure, R. J., \& Jarvis, M. J. 2002, MNRAS, 337, 109

Oke, J. B. 1990, AJ, 99, 1621

Sbarufatti, B., et al. 2005, AJ, 129, 559

Sbarufatti, B., et al. 2006, AJ, 132, 1

Schneider, D. P., et al. 2007, AJ, 134, 102

Shen, Y., et al. 2008, ApJ, 680, 169

Shetrone, M., et al. 2007, PASP, 119, 556

Tody, D. 1986, Proc. SPIE, 627, 733

Tsuzuki, et al. 2006, ApJ, 650, 57

Valdes, F. 1992, in ASP Conf. Ser. 25, Astronomical Data Analysis Software and Systems I, ed. D. M. Worrall, C. Biemesderfer, \& J. Barnes (San Francisco, CA: ASP), 398

Vestergaard, M., \& Osmer, P. S. 2009, ApJ, 699, 800 